\title{
PRODUCTOS FORESTALES NO MADERABLES: IMPORTANCIA E IMPACTO DE SU APROUECHAMIENTO
}

Palabras clave: productos forestales no maderables (PFNM) impacto ecológico, poblaciones, sostenibilidad, manejo.

Key Words: non-timber forest products (NTFP) ecological impact, population, sustainability, management.

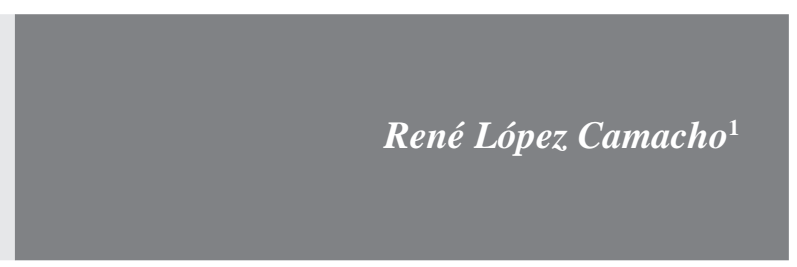

\section{RESUMEN}

Se reconoce que los productos forestales no maderables (PFNM) son importantes para el bienestar de muchas comunidades rurales y contribuyen a los procesos de conservación de los bosques tropicales. Como una aproximación al conocimiento del impacto ocasionado por su aprovechamiento, y a partir de la revisión de varios estudios, el presente artículo expone las consecuencias de esta actividad en diferentes niveles ecológicos (individuo, población y ecosistemas) y las formas y los efectos del aprovechamiento, presentando el estado actual y las tendencias de investigación que conlleven a un uso y manejo sostenible de los PFNM. Se concluye que es prioritario el estudio de estos productos de una manera sistémica, que debe ir más allá del contexto ecológico y biológico, donde se involucren los componentes sociales, económicos, culturales y políticos, logrando el desarrollo de modelos predictivos que garanticen el no deterioro de estos recursos.

\section{ABSTRACT \\ It is recognized that non-timber forest products (NTFP) are important for the well-being of many ru- ral communities and contribute to the conservation of tropical forests. As an approach to understanding the impact of harvesting and from the review of se- veral studies, this paper sets out the consequences}

of this activity in different ecological levels (individuals, populations and ecosystems) and the ways and purposes of harvesting, introducing the current status and trends of research leading to a sustainable use and management of NTFP. We conclude that the study of these products in a systemic way is a priority, and that ir must go beyond the biological and ecological context, and involve the social, economic, cultural and political contexts through the development of pattern-predictive models, which could ensure the non-reduction of these resources.

\section{INTRODUCCIÓN}

Los bosques tropicales, en términos de diversidad ecosistémica, riqueza biológica y oferta de bienes y servicios ambientales, constituyen una parte esencial de los medios de subsistencia de las comunidades que allí habitan, tanto en aspectos de recolección, como de consumo de productos vegetales naturales (Carpentier et al. 2000, Dovie 2003, Ticktin 2005).

Pese a que el aprovechamiento de los productos del bosque es una actividad que ha sido desarrollada durante miles de años (Padoch 1992, Godoy \& Bawa 1993), sólo en los últimos treinta años los productos forestales no maderables (PFNM) se han constituido en objeto de interés a nivel mundial, debido, entre otras razones, a la preocupación que

1 Universidad Distrital Francisco José de Caldas, Facultad de Medio Ambiente y Recursos Naturales, Bogotá, Colombia, rlopezc@udistrital.edu.co. 
se dio a finales de los ochenta en torno al medio ambiente, la deforestación y el bienestar de las comunidades (FAO 1991). Recientemente, este interés ha girado en torno a la búsqueda de opciones para el alivio de la pobreza y la conservación del ambiente (Homma 1992, Redford 1992, Dove 1993, Redford \& Sanderson 2000, Schwartzman et al. 2000, Salafsky \& Wollenberg 2000, Campos et al. 2001, Arnold \& Ruiz-Pérez 2001), a través de estrategias de diversificación de las distintas actividades productivas que mejoren los ingresos de las comunidades locales, provean seguridad alimentaria y ayuden a la conservación de la diversidad biológica y cultural (De la Peña \& Illsley 2001).

Las perspectivas de aprovechamiento que hoy presentan los PFNM pueden ocasionar su sobreutilización o sobreexplotación, conllevando a una declinación de las poblaciones nativas con fuertes implicaciones ecológicas y económicas. Neumann $\&$ Hirsch (2000) documentan a partir de varios estudios de caso que a medida que aumenta la presión sobre el recurso y el número de actores implicados en su comercialización, hay un desconocimiento de criterios para su aprovechamiento y manejo sobre una base sostenible.

Este artículo abarca una revisión y reflexión del impacto ecológico generado en el aprovechamiento de los PFNM, con énfasis en lecciones aprendidas en los trópicos, presentando los aspectos necesarios para el estudio y la implementación de métodos de evaluación de su aprovechamiento desde una perspectiva de sostenibilidad y conservación de los bosques; en esta medida, documenta el efecto del aprovechamiento sobre las especies y poblaciones de plantas vasculares para el consumo humano o industrial $y$, al final, se recoge una serie de lineamientos de investigación, necesarios para abordar, desde una perspectiva investigativa, el tema de los PFNM.

A pesar de la extensa literatura en PFNM, existen vacíos metodológicos de evaluación y monitoreo del impacto ocasionado por el aprovechamiento de las poblaciones naturales y esquemas de valoración de oferta de estos recursos en los diferentes ecosistemas, que permitan un manejo adecuado de ellos (Sheil \& Wunder 2002).

\section{DEFINICIÓN DE LOS PFNM}

Durante muchos años, los PFNM, también conocidos como Non-Timber Forest Products (NTFP), fueron considerados productos secundarios o menores del bosque (Arnold \& Ruiz-Pérez 2001). El término fue empleado por primera vez por De Beer \& McDermott (1989), quienes propusieron la siguiente definición: “...los productos forestales no maderables abarcan todos los materiales biológicos diferentes a la madera, que se extraen de los bosques para uso humano".

En la práctica, dependiendo del propósito de la investigación y el contexto de los estudios (local, nacional o internacional), las generalizaciones acerca de los PFNM son confusas y han dado como resultado diferentes interpretaciones. Sin embargo, es claro que los PFNM hacen referencia a un sector de la producción forestal cuya definición hoy cuenta con cerca de treinta connotaciones distintas (Nepstad \& Schwartzman 1992, Belcher 2003), donde un componente común en su definición es que excluyen la madera, y que el producto, beneficio o servicio que prestan, debe proceder de un bosque o de árboles situados en otros terrenos (Wong et al. 2001).

De acuerdo con la Organización de la Naciones Unidas para la Agricultura y la Alimentación (FAO, por sus siglas en inglés, 2007), los productos forestales no madereros son bienes de origen biológico, distintos de la madera, derivados del bosque, de otras áreas forestales y de los árboles fuera de los bosques. LOS PFNM pueden recolectarse en forma silvestre o producirse en plantaciones forestales o sistemas agroforestales. Estos productos en su gran mayoría de origen vegetal son utilizados como alimentos y aditivos alimentarios (semillas comestibles, hongos, frutos, condimentos, aromatizantes), fibras, instrumentos o utensilios, resinas, gomas, y otros usados con fines medicinales, cosméticos o culturales.

Un término que también es bastante empleado corresponde a Non-Wood Forest Products (NWFP), para hacer referencia a los productos no leñosos. Burgener \& Walter (2007) diferencian los NTFP de los NWFP, aclarando que "El término NWFP excluye todas las materias primas leñosas. En consecuencia, 
la madera, virutas, carbón y leña, así como la madera empleada en la elaboración de herramientas, equipo doméstico y tallas, están excluidos".

También los sistemas para la clasificación de los PFNM son variados, desde los que usan agrupaciones de formas de vida (i.e. el bambú, ratán, palmas) o categorías funcionales (i.e. plantas medicinales y aromáticas, carne de animales silvestres, talla de madera, etc.), hasta los basados en categorías de uso y empleados en estudios de tipo etnobotánico como medicinales, alimenticias, artesanales y tóxicas, entre otras (Wong et al. 2001). Si bien muchas de estas clasificaciones han sido útiles en la organización y comprensión ecológica del tema, resultan a menudo inadecuadas para efectuar seguimientos a largo plazo y poder conjugar las dinámicas y evoluciones que presentan éstos en los mercados y sus implicaciones en las actividades de conservación o, incluso, sobre las consecuencias del uso y la gestión de los recursos, de ahí la necesidad de desarrollar sistemas de clasificación que permitan ayudar en el registro de información y servir de base para la comprensión sobre los usos y la demanda de productos y ayudar a la elaboración de metodologías de seguimiento de estos recursos (González 2003).

\section{IMPORTANCIA DE LOS PFNM}

Los bosques fueron estudiados durante mucho tiempo bajo el enfoque de obtención de madera con fines industriales; incluso algunas directrices en la ordenación de los bosques tropicales tenían como objetivo la producción de madera (FAO 1999). Afortunadamente los bosques han cobrado un renovado interés como fuente de diversos productos distintos a la madera y como generadores de múltiples servicios para las poblaciones locales (de Beer \& McDermott 1989, Nepstad \& Schwartzman 1992, Plotkin \& Famolare 1992, Ros-Tonen \& Wiersum 2005).

En la actualidad existen más de 4.000 especies empleadas como PFNM en actividades de subsistencia y actividades culturales, muchas de las cuales han alcanzado cadenas de comercialización (Iqbal 1993, SCDB 2001), y cerca de 150 productos de importancia en términos de comercio internacional, tales como miel, goma arábiga, roten, bambú, corcho, nueces, hongos, resinas, aceites esenciales, medicamentos y principios activos, entre otros (FAO 2008).

Los PFNM han jugado un importante papel en muchos proyectos de conservación y desarrollo, basados en el supuesto de que soportan la producción y el comercio y ayudan a mejorar las condiciones de vida, sin comprometer el ambiente (Myers 1988, Balick \& Mendelsohn 1992, Nepstad \& Schwartzman 1992, Panayotou \& Ashton 1992, Plotkin \& Famolare 1992, Lawrence 2003). Este supuesto se basa en que:

1. La producción y comercialización de los PFNM pueden proveer opciones atractivas económicamente para las comunidades (colonos, campesinos e indígenas) ayudando a incrementar sus ingresos y ofreciendo una oportunidad de desarrollo.

2. La producción de PFNM es más favorable para el uso de los bosques tropicales que otros usos alternativos de la tierra, siendo un paradigma en la valoración y conservación de los bosques tropicales.

3. Incrementando el valor de los PFNM obtenidos por la población local, se aumentan los incentivos para la conservación del bosque, contribuyendo en la prevención del cambio de uso de la tierra con otros fines.

4. La recolección de PFNM es más benigna que el aprovechamiento de la madera u otros usos del bosque, logrando a su vez una base para el manejo forestal sostenible.

Durante muchos años algunas comparaciones optimistas acerca de los PFNM llegaron a sugerir que el total de los valores se acercaba o superaba el valor de la madera misma obtenida en los bosques tropicales (Peters et al. 1989), sin embargo, otras comparaciones más realistas sugieren que los PFNM presentan un valor neto más bajo (Godoy et al. 2000, Sheil \& Wunder 2002). Recientemente, Vantomme (2007) presentó los ingresos generados por los NWFP a nivel mundial, cuantificando un total de 4.720 millones de dólares tan sólo para el año 2005 (Tabla 1). En Colombia el sector de exportaciones en artesanías logró un valor de 19 millones de dólares para el año 2000, donde el 25\% corresponde a artesanías elaboradas 
Tabla 1. Valor de ingreso de NWFP en el 2005 (en millones de dólares).

\begin{tabular}{lcccccc}
\hline Región & Alimento & Carne de monte & Exudados & Plantas ornamentales & Otros & Total \\
\hline Asia & 818 & --- & 316 & --- & 597 & 1.731 \\
Europa & 382 & 617 & ---- & 344 & 461 & 1.805 \\
África & 4 & 3 & 42 & ---- & 847 & 897 \\
Suramérica & 96 & 5 & 2 & --- & 95 & 197 \\
Norte y Centro América & 34 & ----- & 15 & --- & 22 & 72 \\
Oceanía & ---- & ----- & ---- & -- & 18 & 19 \\
Total mundial & 1.335 & 624 & 376 & 344 & 2.041 & 4.720 \\
\hline
\end{tabular}

Fuente: Vantomme (2007).

en materiales como cueros, tejidos, semillas y madera tallada (Gómez \& Ortega 2007).

Si bien se generan recursos por la venta de estos productos, algunos estudios han mostrado que esta actividad tiende a proporcionar un bajo nivel de ingresos para los sectores más pobres de las comunidades, en lugar de representar una ventaja socioeconómica, llegando en algunos casos a perpetuar la pobreza en vez de aliviarla (Neumann \& Hirsch 2000, Belcher 2003).

El carácter selectivo de la demanda del mercado y el valor de uso de los recursos dentro de los bosques por parte de las comunidades hacen que, en la práctica, el aprovechamiento de los PFNM pueda ser no sostenible (Arnold \& Ruiz-Pérez 2001), ya que las fuerzas del mercado pueden generar conflictos de intereses entre aquellos pobladores que utilizan los recursos forestales para la subsistencia y aquellos que buscan la generación de ingresos en corto tiempo.

\section{IMPACTOS DEL}

\section{APROVECHAMIENTO DE PRODUCTOS FORESTALES NO MADERABLES}

La demanda y el consumo de diversos productos del bosque vienen provocando su agotamiento a un ritmo alarmante, siendo una de las causas subyacentes de la pérdida de biodiversidad (Broad et al. 2003).

A nivel mundial quizá el ejemplo más relevante de esta situación lo constituye el cerezo africano,
Prunus africana (Hook. f.) Kalm., cuya corteza ha sido utilizada para el tratamiento de la hiperplasia benigna de próstata y otros trastornos, siendo Camerún, Madagascar, Guinea Ecuatorial y Kenya los principales exportadores de este recurso. Las exportaciones de corteza seca, en el año 2000, se estimaron entre 1.350 y 1.525 toneladas métricas, valor muy por debajo del alcanzado en el año de 1997 que fue de 3.225 toneladas. La sobreexplotación de este recurso llevó a que en el año 2000 la empresa Plantecam, el mayor exportador de corteza en África, cerrara su fábrica. La especie se encuentra actualmente incluida en el apéndice II de CITES, dentro de la categoría "en peligro de extinción" (Stewart 2003).

En Colombia, la recolección excesiva, con fines comerciales y ornamentales, de varios grupos de plantas como orquídeas, zamias y palmas, ha propiciado que varias especies se encuentren en alguna categoría de amenaza. La explotación de orquídeas de los géneros Anguloa, Cattleya y Cycnoches, es un factor que incide en las poblaciones naturales, llevándolas a diferentes niveles de amenazas (Cálderon-Sáenz 2007). Casos similares se documentan para varias especies de zamias, principalmente Z. encephalartoides y Z. wallisii, de las cuales se ha realizado extracción ilegal de tubérculos y semillas con fines comerciales (Galeano et al. 2005), y a nivel de palmas, las que presentan amenaza por sobreexplotación del recurso son la palma estera (Astrocaryum malybo) y las palmas de cera (Ceroxylon spp.), cuyas hojas se extraen para la elaboración de ramos durante las temporadas de Semana Santa (Galeano \& Bernal 2005). 
El aprovechamiento de los PFNM es diverso y tiene varios niveles de incidencia, desde el genético, de individuos, poblaciones, comunidades y ecosistemas (Hall y Bawa 1993, Peters 1994). No obstante, se pueden identificar dos grandes clases de impactos ecológicos originados en el aprovechamiento y manejo de un recurso biológico:

- Impactos sobre las especies en sí, con los efectos sobre tamaño de población, distribución y composición genética de la población como resultado del aprovechamiento selectivo o de una manipulación genética deliberada.

- Impactos sobre el ecosistema, los cuales están relacionados con las actividades del aprovechamiento y manejo y la decisión acerca del uso futuro de los bosques donde se efectúa el aprovechamiento (Evans 1993).

El problema se vuelve complejo si tenemos presente que una especie vegetal puede estar representada en diferentes tipos de ecosistemas, presentando diferentes grados de abundancia, y que cada uno de estos ecosistemas puede exhibir diferentes grados de intensidad de uso por las comunidades que en ellos habitan.

El impacto ecológico sobre la especie aprovechada es, en la mayoría de casos, desconocido y ha dado lugar a enérgicos debates sobre el valor del aprovechamiento de los PFNM frente a la conservación y al bienestar social y económico de las zonas rurales y de las poblaciones marginadas (Panayotou \& Ashton 1992, Dove 1993, Arnold \& Ruiz-Pérez 2001, Marshall et al. 2003).

De otra parte, los estudios poblacionales constituyen una forma de evaluar el impacto producido por el aprovechamiento de los PFNM, posibilitando la generación de modelos para el manejo sostenible de las poblaciones objeto de extracción (Vásquez \& Gentry 1989, Shankar et al. 1996, 1998, Lambert 1998, Bhatnagar 2002, Ticktin 2004); sin embargo, se reconoce que son muchas las interrelaciones a tener en cuenta para lograr una buena aproximación a un aprovechamiento sostenible, siendo necesario contemplar aspectos ecológicos, biológicos, socioculturales y económicos del lugar donde se aprovecha el recurso (Duivenvoorden et al. 1999, López \& Cavelier 2007).

Para el entendimiento de las dinámicas poblacionales y el impacto sobre su aprovechamiento, se han empleado modelos de matrices, principalmente los llamados modelos de poblaciones estructuradas, que contienen categorías de individuos agrupados por edad o tamaño (Tuljapurkar \& Caswell 1997) y se enfocan a responder preguntas como cuáles son las categorías de edad que pueden estar más comprometidas o encontrarse más afectadas por el aprovechamiento, cómo se afectan las tasas demográficas de la población por diversas intensidades de aprovechamiento y cómo se comporta la población a través del tiempo si se mantiene constante una tasa de aprovechamiento.

Un aspecto diferencial relevante entre los individuos que componen una población es que coexisten individuos de diversas edades, quienes exhiben comportamientos demográficos totalmente distintos; aspectos como fertilidad y tasa de mortalidad no presentan una misma probabilidad en cada categoría de edad y varían dependiendo de la población estudiada. Sin embargo la edad no es siempre la que va a determinar las características reproductivas o de supervivencia de un individuo, sino que puede ser su tamaño o su estadio dentro del ciclo vital, de ahí que sea importante estructurar las poblaciones en estadios o en tamaños (Piñol \& Martínez-Vilalta 2006).

Zuidema (2000), mediante el empleo de estos modelos, ilustra cómo el aprovechamiento de los PFNM puede influenciar la dinámica de la población a partir de un ciclo de vida generalizado de cinco categorías: semillas, plántulas, juvenil, adulto joven y adulto viejo (Figura 1).

De acuerdo con este modelo, los impactos pueden ser directos para el caso donde se aprovechan semillas, frutas, nueces, palmito (diagrama a y b) o no puede cuantificarse en forma directa (diagrama c). Para la situación (a) hay un alto impacto en la primera categoría poblacional, lo cual va a incidir en toda la población; se efectúa aprovechamiento de semillas y frutos. En la situación (b) el impacto directo radica en los adultos jóvenes y adultos viejos, donde 
se sacrifica el individuo, es el caso del aprovechamiento de muchas hierbas medicinales en donde se colecta todo el individuo o en el aprovechamiento del palmito. Finalmente, en la situación (c), donde el impacto no es directamente cuantificable (extracción de látex, resinas, gomas, cortezas, etc.), se efectúa un aprovechamiento generalmente sobre adultos jóvenes y adultos viejos sin sacrificarlos. En estos casos el impacto puede ser medido por ejemplo en la producción de frutos o flores o en la producción de algún otro tipo de órgano de la planta.

El uso de estos modelos estructurados permite identificar qué edades o categorías son más críticos desde el punto de vista demográfico y, por tanto, en cuáles concentrar los esfuerzos de gestión. Sin embargo en la aplicación práctica de estos modelos para obtención de PFNM es necesario considerar otros factores como aquellos que tienen que ver con la competencia intraespecífica, estocasticidad ambiental y factores socioculturales del aprovechamiento.

\section{IMPACTOS SOBRE LOS INDIVIDUOS}

Muchas plantas exhiben un número de rasgos biológicos que permiten potencializar respuestas positivas para el aprovechamiento sostenible (Siebert 2004), otras por el contrario tienen respuestas muy sensibles al aprovechamiento; por consiguiente, los estudios ecológicos sobre los PFNM no son

\section{Impacto directo}

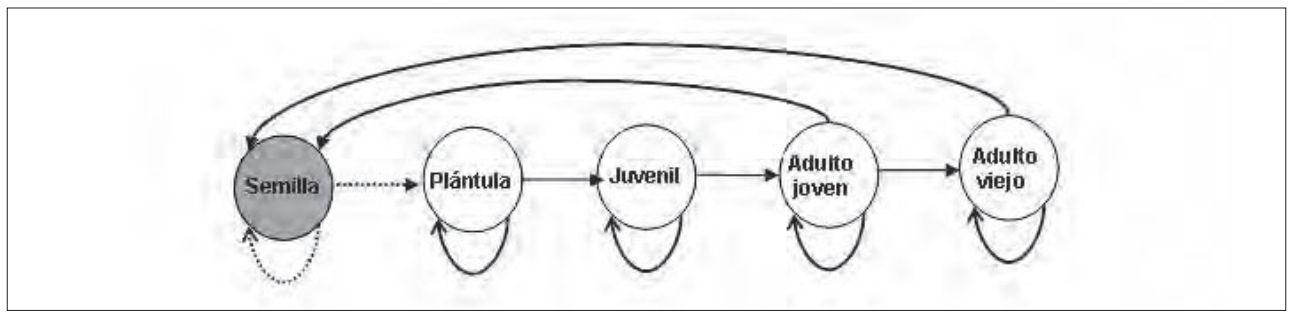

(a)

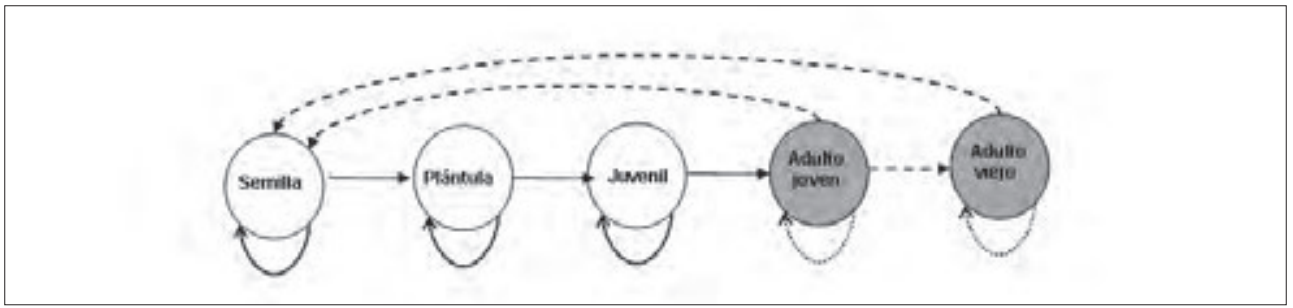

(b)

Impacto no directamente cuantificable

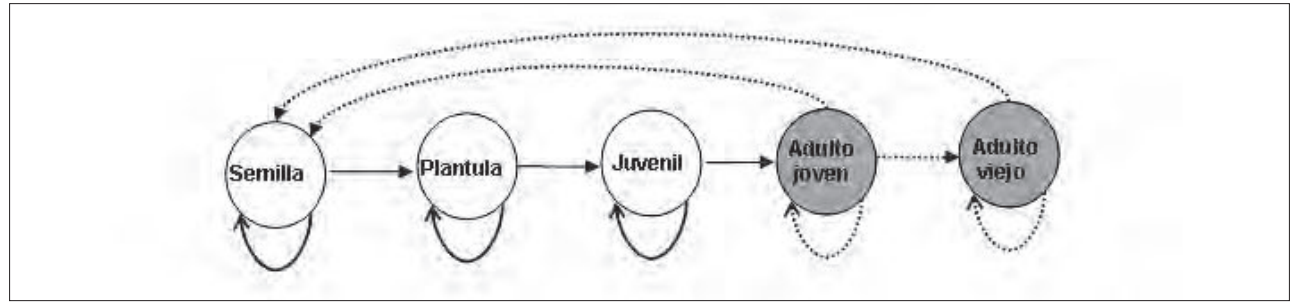

(c)

Figura 1. Influencia de la parte extraída de plantas en la dinámica poblacional. Los círculos sombreados y las líneas discontinuas indican aquellas categorías en donde se aprovechan los individuos y que requieren mayor atención para ser cuantificados en el aprovechamiento de la especie. Las líneas con puntos más finos indican sobre qué otros grupos indirectamente se tendrán impactos. Fuente: Zuidema (2000). 
fácilmente generalizados, pues se reporta diferente grado de impacto dependiendo de la especie y la parte aprovechada. Algunas especies parecen ser muy tolerantes al aprovechamiento (Zuidema \& Boot 2002, Ticktin et al. 2002), otro grupo de plantas por el contrario muestran impactos negativos altos (Olmsted \& Alvarez-Bullya 1995, Zuidema 2000, Soehartono \& Newton 2001).

En el aprovechamiento de las especies, los efectos sobre la demografía de plantas dependen de la parte de la planta aprovechada (Olmsted \& AlvarezBuylla 1995, Pfab \& Scholes 2004, Ticktin 2004). Ayuk et al. (1999) y Dovie et al. (2002), tomando como referencia la clasificación de los PFNM según la parte usada, presentan cuatro grandes categorías: 1) frutos carnosos, nueces y aceite de semillas; 2) exudados de plantas como látex, resinas y néctar floral; 3) estructuras vegetativas como yemas apicales, bulbos, hojas, tallos, cortezas y raíces, y 4) tallos pequeños, bastones y "palos", aprovechados para vivienda, cercas, leña, y artesanía.

Cada una de estas categorías incluye un gran número de especies de plantas y cada una de las partes presenta una diversidad de patrones de crecimiento y formas de vida que pueden tener diversa respuesta al aprovechamiento (Ticktin 2004) y que deben considerarse para un manejo sostenible.

La Tabla 2 presenta algunas especies vegetales de interés en la obtención de PFNM en Colombia, indicando la parte aprovechada, su hábito y categoría de uso.

El hábito de la especie, el lugar donde se desarrolla, la parte usada y la intensidad del aprovechamiento, son aspectos relevantes a tener en cuenta para medir su impacto. Ticktin (2004) reporta que las hierbas perennes en regiones templadas presentan un alto grado de vulnerabilidad al aprovechamiento; la única hierba que soporta altos niveles de aprovechamiento es Aechmea magdalenae, empleada en la obtención de fibra en México. Esta diferencia se atribuye a que la especie se desarrolla en climas más favorables para su crecimiento, como son los de las regiones tropicales.

Ghimire et al. (2005) analizan el efecto del aprovechamiento del rizoma en la ecología de las especies medicinales Nardostachys grandiflora DC. (Valerianaceae) y Neopicrorhiza scrophulariiflora (Pennell) D.Y. Hong (Scrophulariaceae), bajo dos grandes enfoques: el aprovechamiento efectuado por los médicos tradicionales, quienes cosechan las plantas de manera selectiva para uso local en atención en salud, y otro grupo, colectores comerciales, que efectúan un aprovechamiento no selectivo y con una mayor intensidad y fines comerciales. Ellos determinan que hay un efecto positivo sobre la densidad de las poblaciones cuando éstas se aprovechan con bajos niveles de recolección, método empleado por los amchi (médicos tradicionales). Al aumentar los niveles de recolección, las tasas de reclutamiento y de supervivencia de las especies disminuyen, método empleado por los colectores comerciales. Concluyen que el aprovechamiento debe tener en cuenta la adopción de patrones de cosecha, las formas de vida vegetal y las pautas de crecimiento, para poder disminuir su impacto.

La tolerancia al aprovechamiento también varía con la historia de vida de las especies. Por ejemplo, las poblaciones de hierbas perennes pueden soportar tasas más altas de aprovechamiento que las poblaciones de árboles. Estos datos están soportados bajo análisis del modelo de elasticidad de especies de árboles que indican que la supervivencia de la mayor etapa de clases de edad contribuye más al crecimiento de la población, mientras que las semillas contribuyen en menor grado (Peters 1991, Olmsted \& Alvarez-Buylla 1995, Ratsirarson et al. 1996, Stewart 2001). Algunos estudios sugieren que muchos árboles no son capaces de resistir bajas tasas de aprovechamiento, conduciendo en muchos casos a la mortalidad de los individuos, por lo que es necesario tener en cuenta que la cosecha de los PFNM como tallos, corteza y partes apicales puede implicar meristemos que inciden a largo plazo en la disminución de la población y en su persistencia en el largo plazo (Vásquez \& Gentry 1989, Sinha \& Bawa 2002).

\section{IMPACTO SOBRE LAS POBLACIONES Y ECOSISTEMAS}

Zuidema (2000) y Svenning \& Macia (2002) han comprobado cómo el aprovechamiento de hojas de palmas del sotobosque no tolera altos niveles de 
Tabla 2. Especies de interés en la obtención de PFNM, categoría de uso y hábito.

\begin{tabular}{|c|c|c|c|c|}
\hline Parte aprovechada & Especie & Nombre común & Categoría de uso & Hábito \\
\hline \multirow{7}{*}{ Planta entera } & Petiveria alliacea & Anamú & Medicinal & Hierba \\
\hline & Taraxacum officinale & Diente de león & Medicinal & Hierba \\
\hline & Calamagrostis effusa & Paja blanca & Artesanal & Hierba \\
\hline & Uncaria guianensis /Uncaria tomentosa & Uña de gato & Medicinal & Bejuco \\
\hline & Lippia alba & Pronto alivio & Medicinal & Hierba \\
\hline & Sambucus nigra & Saúco & Medicinal & Arbusto \\
\hline & Gynerium sagitatum & Caña flecha & Artesanal & Hierba \\
\hline \multirow{10}{*}{ Semillas y frutos } & Phytelephas seemanii & Tagua & Artesanal & Palma \\
\hline & Canna indica & Achira, achirilla & Artesanal & Hierba \\
\hline & Coix lacryma-jobi & Lágrimas de San Pedro & Artesanal & Hierba \\
\hline & Sapindus saponaria & Chambimbe, jaboncillo & Artesanal & Árbol \\
\hline & Abrus precatorius & Pionia, chocho & Artesanal & Hierba \\
\hline & Ormosia spp. & Chocho & Artesanal & Árbol \\
\hline & Mucuna spp. & 0jo de buey & Artesanal & Bejuco \\
\hline & Juglans neotropica & Cedro nogal & Artesanal & Árbol \\
\hline & Entada gigas & Congolo & Artesanal & Bejuco \\
\hline & Genipa americana & Huito & Artesanía & Árbol \\
\hline \multirow{3}{*}{ Hojas } & Geonoma orbignyana & Palmilla & Construcción & Palma \\
\hline & Geonoma deversa & San Pablo & Construcción & Palma \\
\hline & Carludovica palmata & Palma iraca & Artesanía & Hierba \\
\hline \multirow{2}{*}{ Inflorescencias } & Heliconia spp. & Platanillos & Ornamental & Hierba \\
\hline & Calathea spp. & Platanillos & Ornamental & Hierba \\
\hline \multirow{3}{*}{ Látex y resinas } & Croton lechleri & Sangre de drago & Medicinal & Árbol \\
\hline & Ficus insipida & Higuerón & Medicinal & Árbol \\
\hline & Hymenaea courbaril & Algarrobo & Artesanía & Árbol \\
\hline \multirow{4}{*}{ Corteza-Raíces } & Cinchona spp. & Quina & Medicinal & Árbol \\
\hline & Maytenus laevis & Chuchuhuaza & Medicinal & Árbol \\
\hline & Ficus spp. & Yanchamas & Artesanía & Árbol \\
\hline & Philodendron spp. & Bejuco tripa de perro & Artesanía & Bejuco \\
\hline
\end{tabular}

cosecha, por lo menos en condiciones de bosques primarios, mientras que varias de las palmas del dosel y de áreas abiertas pueden soportar mayores tasas de aprovechamiento en la cosecha de la hoja (Fong 1995, Ratsirarson et al. 1996).

De otra parte, las tasas de crecimiento demográfico y las respuestas al aprovechamiento pueden variar considerablemente a lo largo de gradientes climáticos y de suelos. Otros estudios adelantados por Shankar et al. (1996) y Svenning (2002) han indicado la influencia de diversos factores ambientales sobre el rendimiento de las poblaciones sometidas al aprovechamiento. Por ejemplo, Nantel et al. (1996) estudiaron poblaciones de Panax quinquefolium (ginseng americano) y Allium tricoccum 
(puerro silvestre), mediante una evaluación del impacto del aprovechamiento en ambientes que varían estocásticamente y estimando umbrales de extinción y sus poblaciones mínimas viables; como resultado, encontraron, para ambas especies, que los impulsos de un ambiente que varía de esta manera reduce significativamente los niveles de cosecha sostenibles.

La mayoría de estudios poblacionales han sido desarrollados en el grupo de las palmas. Zuidema (2000), estudiando Euterpe precatoria para obtención de palmito, concluye que la explotación intensiva es incompatible con la historia de vida de la especie debido a su baja disponibilidad de reclutamiento. De otra parte, Bernal (1998) encontró que la tagua (Phytelephas seemannii) tolera altos niveles de aprovechamiento de la semilla, debido a que presenta una alta producción y la mortalidad de los adultos es baja después de haber sido aprovechados.

Estudios sobre el aprovechamiento de hojas de palma han mostrado diferentes respuestas; en algunas se ha visto un incremento en la tasa de producción de hojas, en otras una reducción en la producción de inflorescencias (Endress et al. 2004). Mendoza et al. (1987) encontraron que el aprovechamiento puede incrementar o decrecer la probabilidad de producción de frutos dependiendo de qué hojas sean removidas, y los efectos sobre la mortalidad y el rendimiento reproductivo pueden ser subestimados debido a que el efecto del aprovechamiento puede ser acumulativo, como se comprobó al evaluar la producción de frutos tras cosechar hojas de Chamaedorea radicalis, después de seis años de estudio bajo diferentes intensidades de aprovechamiento (Endress et al. 2006). Rodríguez-Buriticá et al. (2005), analizando la dinámica de la población de Geonoma orbygniana y buscando dar respuesta entre otros aspectos a las características de esta palma para su manejo desde la perspectiva de una aproximación demográfica, encontraron que la especie puede ser especialmente sensible a la explotación intensa, ya que presenta una baja tasa de crecimiento (1.98 hojas/año).

Finalmente, el aprovechamiento de los PFNM incide sobre el funcionamiento de las comunidades y ecosistemas: un ejemplo de esto es el impacto que tiene sobre la presencia, abundancia y diversidad de frugívoros y granívoros. Algunos autores sostienen que el impacto puede ser significativo cuando la mayoría de los frutos colectados para la venta sirven de alimento a los grandes mamíferos y aves, o las frutas, semillas o flores aprovechadas condicionan la presencia y abundancia de frugívoros y granívoros. Moegenburg \& Levey (2002) mostraron que un alto aprovechamiento de los frutos de la palma Euterpe oleracea en la Amazonia brasilera reduce la diversidad de frugívoros aéreos. Galetti \& Aleixo (1998), evaluando el impacto ocasionado por el aprovechamiento de palmitos (Euterpe edulis), concluyeron que éste afecta negativamente la abundancia de grandes aves frugívoras.

\section{FORMAS DE}

\section{APROVECHAMIENTO Y EVALUACIÓN DE SU SOSTENIBILIDAD}

Zuidema (2000) plantea que en la evaluación del aprovechamiento de un producto para determinar si es o no sostenible se deben considerar el individuo (evaluando si el aprovechamiento causa la muerte), la población (considerando un aprovechamiento sostenible si la población no desaparece o si la productividad vista desde la disponibilidad del recurso a aprovechar no disminuye con el tiempo) y el ecosistema (si el aprovechamiento de los recursos no cambia su funcionamiento). De otra parte, Kusters et al. (2006) proponen tres niveles para evaluar la sostenibilidad de la extracción de los PFNM: 1) poblaciones de las especies objeto de aprovechamiento, donde los estudios de caso han sido focalizados en un área particular; 2) uso del suelo de los ecosistemas, con referencia a la zona dentro del área de estudio en que el grupo de especies de PFNM es producido (bosque secundario, bosque inundable, sistema agroforestal, bosque de tierra firme, etc.), y 3 ) nivel regional del paisaje, referido al área de estudio generalmente compuesta por un mosaico de usos de la tierra.

Cada nivel debe relacionarse con un conjunto de indicadores que comprendan aspectos de tipo social y económico. Belcher et al. (2005), implementando indicadores de tipo social al papel que juegan los PFNM 
en la economía local, han logrado la identificación de cinco grandes grupos de comunidades que aprovechan los PFNM: grupo de subsistencia, grupo suplementarios, grupo integrado, grupo de extracción especializado y grupo de cultivos especializados. Cada grupo muestra un claro patrón de relaciones con un amplio rango de otras variables e incide en las formas de impacto del aprovechamiento.

El entendimiento de las formas de extracción local y las prácticas de manejo en el aprovechamiento de los PFNM se encuentran muy correlacionados con el componente socioeconómico e implican entender la ecología de la planta dentro del contexto de cómo es ésta usada localmente (Cunningham 2001, Ticktin 2004). En este aspecto, ha existido un interés creciente en la simulación local de estrategias de manejo para evaluar los niveles de sostenibilidad de extracción de PFNM (Ticktin et al. 2002).

A nivel de algunas técnicas de aprovechamiento y su incidencia en el impacto, los métodos silviculturales toman importancia y validez, ya que algunas prácticas de manejo, como disminución de poblaciones densas o siembra de semillas, pueden resultar en tasas de crecimiento de poblaciones aprovechadas que exceden a aquellas poblaciones que no lo han sido (Ticktin et al. 2002).

Paoli et al. (2001), estudiando la especie Aquilaria malaccensis, encontraron variaciones en las densidades y respuestas a la intensidad de aprovechamiento, concluyendo que los rendimientos por unidad de área pueden mejorar sustancialmente mediante la instrumentación de métodos silviculturales para incrementar la densidad de árboles, aumentando la proporción de árboles que producen madera y reduciendo el intervalo de tiempo entre cosechas sucesivas. Ticktin \& Johns (2002) demostraron que las hojas de Aechmea magdalenae se pueden cosechar mediante dos formas: cortando la hoja más larga o cortando toda la planta, en cuyo caso se consume menos tiempo. Aunque los modelos demográficos ilustran que para el primer caso las plantas proveen más fibra y presentan un crecimiento más rápido que aquellas sujetas al corte total, al aplicar prácticas de manejo de luz y raleos se produce mayor crecimiento y mayor producción de fibra tras cortar toda la planta, lo cual muestra la importancia de combinar las prácticas de manejo con técnicas de aprovechamiento.

\section{RECOMENDACIONES PARA EL MANEJO DE LOS PFNM}

Es evidente que los PFNM han desempeñado y desempeñarán un papel fundamental en la vida de millones de personas, siendo prioritario identificar aquellas variables que generan un impacto (positivo o negativo) sobre estos recursos, buscando el uso sostenible y la conservación de los bosques.

Los estudios de modelos poblaciones relacionados con las historias de vida y las características de las poblaciones objeto de aprovechamiento representan un paso inicial para conocer el efecto del aprovechamiento cuando se planea el manejo de especies; sin embargo, estos estudios requieren ser complementados con otras áreas de conocimiento como son los componentes sociales, políticos y culturales en los cuales se encuentran enmarcados los bosques de donde se obtienen estos recursos.

Si bien se extraen PFNM de una enorme variedad de especies de plantas (FAO 2008), la mayor parte de la investigación en los niveles de individuos y población se ha concentrado en un número limitado de formas de vida y partes de las plantas. Muchos de los estudios se centran en palmas y son muy pocos los orientados a formas de vida como lianas y bejucos. Así mismo, gran parte de las investigaciones adelantadas han examinado los efectos del aprovechamiento sobre frutas, semillas y hojas, dejando de lado investigaciones en exudados vegetales tales como gomas, resinas y oleorresinas, así como cortezas, raíces y bulbos, los cuales representan una gran proporción de la naturaleza comercial cosechada de los PFNM (Vantomme et al. 2002).

De otra parte, una falencia que presentan los estudios sobre el impacto del aprovechamiento de los PFNM es que la mayoría de ellos se han efectuado a corto plazo, esto es, menos de dos años (Nakazono et al. 2004, Ticktin 2005). La revisión adelantada por Ticktin (2004) encontró que tan sólo el 10\% de las poblaciones de PFNM presenta estudios supervisados 
para más de tres años, lo que dificulta predecir el impacto demográfico a largo plazo y determinar los niveles de aprovechamiento sostenible, especialmente en especies de larga vida o en aquellos casos en los que el efecto del impacto sobre la población puede irse acumulando. Por lo tanto, se requiere el desarrollo y la implementación de diseños experimentales que permitan describir y determinar los impactos del aprovechamiento sobre las especies de interés, de tal manera que suministren información sobre los efectos del aprovechamiento en aspectos como tasas de supervivencia, crecimiento y reproducción y, para ello, el establecimiento de parcelas permanentes es una alternativa para evaluar e implementar modelos poblacionales que posibiliten el entendimiento de estos procesos (Vallejo et al. 2005).

Belcher et al. (2005) proponen un método para el análisis divergente de los PFNM a partir de la comparación de 61 casos de producción comercial en África, Asia y América Latina, generando un conjunto de descriptores organizados en categorías (desde la producción hasta el sistema de consumo), con el que concluyen que es importante tener en cuenta en el desarrollo de programas de aprovechamiento de PFNM aspectos como:

- La producción comercial de los PFNM debe estar integrada con otras actividades económicas.

- Una limitante importante radica en que los pobres tienen poco acceso a los mercados, existe insuficiencia de capital humano y productivo, y muchas veces no hay presencia de instituciones, lo que conlleva a un escaso poder de negociación en estas zonas.

- Algunos PFNM pueden ofrecer el potencial para crear empleo y oportunidades de generación de ingresos, pero la realización de este potencial requerirá de inversiones en otras áreas.

- La inversión en PFNM puede ayudar a mejorar la capacidad y obtener un mejor acceso a los recursos; pero puede ser que condiciones externas como fuerzas del mercado o costos de oportunidad hagan que la gente prefiera otras actividades diferentes a la producción de PFNM.

Todas estas implicaciones en torno al aprovechamiento de los PFNM hacen que sea de vital importancia efectuar procesos de valoración de los bienes que representan estos productos en los bosques tropicales. Estas valoraciones deberán influir en quienes toman las decisiones, encargados de la formulación y determinación de políticas y administración de los recursos naturales, con el fin de garantizar la sostenibilidad en el aprovechamiento de los PFNM.

Por ello, desde el contexto ecológico, para aprovechar y conservar de forma efectiva las poblaciones de PFNM, al menos tres preguntas deben ser respondidas: ¿cuáles son los impactos ecológicos del aprovechamiento?, ¿cuáles son los mecanismos subyacentes a estos impactos? y ¿qué tipos de prácticas de manejo y técnicas de aprovechamiento pueden mitigar los impactos negativos o promover impactos positivos?

A continuación se sugieren algunos aspectos relacionados con la investigación en PFNM y sus implicaciones ecológicas:

- Los PFNM deben ser observados como un sistema en donde cada compartimiento es diferente, ya que depende de la variación en la historia de vida de la especie, las partes de plantas aprovechadas y los efectos de alteración ambiental del aprovechamiento.

- La implementación de diversas técnicas de manejo silvicultural y de gestión pueden ser efectivas para disminuir los impactos negativos de algunos tipos de aprovechamiento.

- Es importante evaluar el desarrollo de técnicas de extracción con bajo impacto en aquellas especies que presentan potencial para la obtención de corteza, látex, resinas y gomas, entre otros, buscando disminuir la mortalidad de los adultos.

- En los casos en los que la mortalidad de los adultos sea un requisito necesario como parte del aprovechamiento, es vital el mantenimiento de la regeneración y la implementación de prácticas como el enriquecimiento y la protección de plántulas, el mantenimiento de condiciones adecuadas para la germinación y el crecimiento, y tener presente que el desarrollo de plantaciones o cultivos mixtos es necesario a largo plazo para el desarrollo de una producción sostenida. 
- Las formas de respuesta al aprovechamiento varían significativamente entre las especies y van a estar ligadas a las estrategias de manejo por parte de las comunidades locales que aprovechan estos productos.

- Es necesario buscar herramientas que permitan tener una mayor regulación sobre el aprovechamiento, facilitando las labores de las autoridades ambientales y entes gubernamentales y evitando en muchos casos el choque de intereses.

- Los estudios que se adelanten deberán involucrar las escalas espacial y temporal, logrando discernir sobre los posibles impactos de acuerdo con los ecosistemas involucrados, y deberá implementarse una estrategia que permita el monitoreo de los diversos PFNM en cada uno de ellos a través del establecimiento de parcelas permanentes.

Los usuarios de recursos, entes investigativos, organismos reguladores, organizaciones no gubernamentales, responsables políticos y todos los demás interesados, deben ser consecuentes con la necesidad de seguir haciendo hincapié en el importante papel de los PFNM, abogando por la adopción de medidas relacionadas con su comercio, conservación y uso sostenible. Finalmente, todo actor relacionado con los PFNM debe entender que para lograr la sostenibilidad del uso de un recurso las tasas de recolección no deben exceder la capacidad de las poblaciones para sustituir a los individuos extraídos, y además reconocer que estos productos se mueven bajo una diversidad de variables que pueden incidir en su sostenibilidad.

\section{AGRADECIMIENTOS}

Agradezco especialmente a Rubén Darío Guerrero y Luz Stella Pulido del Ministerio de Ambiente, Vivienda y Desarrollo Territorial, Dirección de Ecosistemas, por su motivación y apoyo en el desarrollo de líneas de investigación en torno a los PFNM. A las investigadoras Nelly Rodríguez y Claudia María Villa del Instituto de Investigación de Recursos Biológicos Alexander von Humboldt, por su lectura y comentarios a este manuscrito. También agradezco a los evaluadores externos, por sus sugerencias en torno al mejoramiento de este escrito.

\section{REFERENCIAS BIBLIOGRÁFICAS}

Anderson, M. K. \& D. L. Rowney. 1999. The Edible Plant Dichelostemma capitatum: its Vegetative Reproduction Response to Different Indigenous Harvesting Regimes in California. Restoration Ecology 7: 231-240.

Angelsen A. \& S. Wunder. 2003. Exploring the Forest-Poverty Link: Key Concepts, Issues, and Research Implications. Occasional Paper 40. CIFOR. Bogor, Indonesia. Pg. 58.

Ankila J. H. 2004. The Ecological Consequences of Managing Forests for Non-Timber Products. Conservation \& Society 2: 211-216.

Anderson, A. B., P. Magee, A. Gely \& M.A. Jardim. 1995. Forest Management Patterns in the Floodplain of the Amazon Estuary. Conservation Biology 9: 47-61.

Arnold, M. \& M. Ruíz-Pérez. 2001. Can NonTimber Forest Products Match Tropical Forest Conservation and Development Objectives? Ecological Economics 39: 437-447.

Ayuk, E. T., B. Duguma, S. Franzel, J. Kengue, M. Mollet, T. Tiki-Manga \& P. Zenkeng. 1999. Uses, Management and Economic Potential of Irvingia gabonensis in the Humid Lowlands of Cameroon. Forest Ecology and Management 113: 1-9.

Balick, M. J. \& R. Mendelsohn. 1992. Assessing the Economic Value of Traditional Medicines from Tropical Rain Forests. Conservation Biology 6: 128-130.

Bhatnagar, P. 2002. Conservation and Trade of Medicinal Herbs: A Study of Safed Musli (Chlorophytum spp.) in Madhya Pradesh. Sustainable Forestry 7: 11-14.

Belcher, B. M. 2003. What isn't an NTFP? International Forestry Review 5 (2): 161-168.

Belcher, B., M. Ruiz-Pérez \& R. Achdiawan. 2005. Global Patterns and Trends in the Use and Management of Commercial NTFPs: Implications for Livelihoods and Conservation. World development 33 (9): 1435-1452. 
Bernal, R. 1998. Demography of the Vegetable Ivory Palm Phytelephas seemannii in Colombia, and the Impact of Seed Harvesting. Journal of Applied Ecology 35: 64-74.

Broad, S., T. Mulliken \& D. Roe. 2003. The Nature and Extent of Legal and Illegal Trade in Wildlife. En: Oldfield, S. (ed.). The Trade in Wildlife. Regulation for Conservation. Earthscan. Londres. Chapter 1.Pg 3-12.

Burgener, M. \& S. Walter. 2007. Trade Measures. Tools to Promote the Sustainable Use of NwFP? An Assessment of Trade Related Instruments Influencing the International Trade in NonWood Forest Products and Associated Management and Livelihood Strategies. NonWood Forest Products Working Document. Forestry Department 6. FAO. Pg. 145.

Calderón-Sáenz, E. (ed.). 2007. Libro rojo de plantas de Colombia. Volumen 6: Orquídeas, primera parte. Serie Libros Rojos de Especies Amenazadas de Colombia. Instituto Alexander von Humboldt y Ministerio de Ambiente, Vivienda y Desarrollo Territorial. Bogotá. Pg. 828.

Campos, J. J., B. Finegan \& R. Villalobos. 2001. Management of Goods and Services from Neotropical Forest Biodiversity: Diversified Forest Management in Mesoamerica, in Conservation and Sustainable Use of Forest Biodiversity Secretariat of the Convention on Biological Diversity (SCBD). Montreal. CBD Technical Series 3: 5-16.

Carpentier, C. L., S. Vosti, \& J. Witcover. 2000. Intensified Production Systems on Western Brazilian Amazon Settlement Farms: Could They Save the Forest? Agriculture, Ecosystems and Environment 82: 73-88.

Casas, A., M. Vásquez, J. L. Viveros \& J. Caballero. 1996. Plant Management Among the Nahua and the Mixtec in the Balsas River Basin, Mexico: An Ethnobotanical Approach to the Study of Plant Domestication. Human Ecology 24: $455-479$

Cunningham, A. B. 2001. Applied Ethnobotany: People, Wild Plant Use and Conservation. Earthscan. Londres. 300 pg.
De la Peña, G. \& C. Illsley. 2001. Los productos forestales no maderables: su potencial económico, social y de conservación. En: Ecológica, 27 de Agosto de 2001. http://www.jornadaunam. $\mathrm{mx} / 2001 / 08 / 27$ eco-a.html

De Beer, J. H. \& M. McDermott. 1989. The Economic Value of Non-Timber Forest Products in South-East Asia. The Netherlands Committee for UICN. Amsterdam. Pg. 197.

Dove, M. 1993. A Revisionist View of Tropical Deforestation and Development. Environmental Conservation 20 (1): 17-24.

Dovie, D. B. K., C. M. Shackleton \& E. T. F., Witkowski. 2002. Direct-Use Values of Woodland Resources Consumed and Traded in a South African Village. International Journal of Sustainable Development and World Ecology 9: 269-283.

Dovie, D. B. K. 2003. Rural Economy and Livelihoods from the Non-Timber Forest Products Trade. Compromising Sustainability in southern Africa? International Journal of Sustainable Development and World Ecology 10: 247-262.

Duivenvoorden, J. F., H. Balslev, J. Cavalier, C. Grández, H. Tuomisto\& R. Valencia. 1999. Non-Timber Forest Plant Resource Assessment in Nw Amazonia. En: Ros-Tonen, M. A. F. (ed.). Seminar Proceedings. NTFP Research in the Tropenbos Programme: Results and Perspectives, 28 January 1999. The Tropenbos Foundation. Wageningen, the Netherlands. Págs. 81-93.

Endress, B. A., D. L. Gorchov \& R. B. Noble. 2004. Non-Timber Forest Product Extraction: Effects of Harvest and Browsing on an Understory Palm. Ecological Applications 14: 1138-1153.

Endress, B. A., D. L. Gorchov \& E. J. Berry. 2006. Sustainability of a Non-Timber Forest Product: Effects of Alternative Leaf Harvest Practices over 6 Years on Yield and Demography of the Palm Chamaedorea radicalis. Forest Ecology and Management 234: 181-191.

Evans, M. I. 1993. Conservation by Comercialization. En: Hladik, C.M., A. Hladik, O. F. Linares, H. Pagezy, A. Semple, Andy \& M. Hadley (eds.). Tropical Forests, People and Food: Biocultural 
Interactions and Applications to Development. MAB Series 13. UnESCO. Paris and Parthenon Publishing Group. Carnforth, UK. Págs. 815-822.

FAO. 1991. Non-Wood Forest Products: the Way Ahead. Organización de las Naciones Unidas para la Agricultura y la Alimentación. Roma. http:// www.fao.org/docrep/T0431E00.htmcontents.

FAO. 1999. Directrices para la ordenación de los bosques tropicales. 1. Producción de madera. Organización de las Naciones Unidas para la Agricultura y la Alimentación. Roma. Pg. 330.

FAO. 2007. Situación de los bosques del mundo 2007. Organización de las Naciones Unidas para la Agricultura y la Alimentación. Roma. http://www.fao.org/docrep/009/a0773s00.htm

FAO. 2008. Productos forestales no maderables. Organización de las Naciones Unidas para la Agricultura y la Alimentación [disponible en: www.fao.org/forestry/site/6388/es].

FSC. 2002. Pacific Coast (USA): Regional Forest Stewardship Standard:Version 7.9[disponible en: http:// www.fscstandards.org/regions/pacific/non forest.html].

Fong, F. W. 1995. Perspectives for Sustainable Resource Utilization and Management of Nipa Vegetation. Economic Botany 46: 45-54.

Freese, C. H. 1998. Wild Species as Commodities: Managing Markets and Ecosystems for Sustainability. Island Press. Washington. Pg. 334.

Galeano, G., R. Bernal, E. Calderón, N. García, A. Cogollo \& A. Idárraga. 2005. Zamias. En: Cálderon, E., G. Galeano \& N. García (eds.). Libro rojo de plantas de Colombia. Volumen 2: Palmas, frailejones y zamias. Serie Libro Rojo de Especies Amenazadas de Colombia.. Instituto Alexander von Humboldt, Instituto de Ciencias Naturales de la Universidad Nacional de Colombia y Ministerio de Medio Ambiente, Vivienda y Desarrollo Territorial. Bogotá. Págs. 387-436.

Galeano, G. \& R. Bernal. 2005. Palmas. En: Calderón, E., G. Galeano \& N. García (eds.). Libro rojo de plantas de Colombia. Volumen 2: Palmas, Frailejones y Zamias. Serie Libro Rojo de
Especies Amenazadas de Colombia. Instituto Alexander von Humboldt, Instituto de Ciencias Naturales de la Universidad Nacional de Colombia y Ministerio de Medio Ambiente, Vivienda y Desarrollo Territorial. Bogotá. Págs. 59-224.

Galetti, M. \& A. Aleixo. 1998. Effects of Palm Heart Harvesting on Avian Frugivores in the Atlantic Rainforest of Brazil. Journal of Applied Ecology 35: 286-293.

Ghimire, K. S., D. McKey \& Y. AumeeruddyThomas. 2005. Conservation of Himalayan Medicinal Plants: Harvesting Patterns and Ecology of Two Threatened Species, Nardostachys grandiflora DC. and Neopicrorhiza scrophulariiflora (Pennell) Hong. Biological Conservation 124: 463-475.

Godoy, R. A. \& K. S. Bawa. 1993. The Economic Value and Sustainable Harvest of Plants and Animals from the Tropical Forest: Assumptions, Hypotheses, and Methods. Economic Botany 47: 215-219.

Godoy, R., D. Wilkie, H. Overman, A. Cubas, G. Cubas, J. Demmer, K. McSweeney \& N. Brokaw. 2000. Valuation of Consumption and Sale of Forest Goods from a Central American Rainforest. Nature 406: 62-63.

Gómez, J.A. \& Ortega. 2007. Biocomercio Sostenible. Biodiversidad y Desarrollo en Colombia. Instituto Alexander von Humboldt. Bogotá. Pg. 138.

González, D.V. 2003. Los productos naturales no maderables (PNNM): Estado del arte de la investigación y otros aspectos. Biocomercio Sostenible. Instituto Alexander von Humboldt. Bogotá.

Gómez, D. J. \& Ortega, S. 2007. Biocomercio sostenible. Biodiversidad y desarrollo en Colombia. Instituto Alexander von Humboldt. Pg. 223.

Hall, P. y K. Bawa 1993. Methods to Assess the Impact of Extractions of Non-Timber Tropical Forest Products on Plant Populations. Economic Botany 47: 234-247.

Homma, A. K. O. 1992. The Dynamics of Extraction in Amazonia: a Historical Perspective. En: Nepstad, D.C. \& S. Schwartzman (eds.). Non-Timber 
Products from Tropical Forests: Evaluation of a Conservation and Development Strategy. Advances in Economic Botany 9: 23-32.

Iqbal, M. 1993. International Trade in Non-Wood Forest Products. An Overview. Food and Agriculture Organization. Roma. http://www.fao. org/docrep/E/5326e00.htm

Kusters, K., A. Ramadhani, B. Belcher \& M. Ruiz-Pérez. 2006. Balancing Development and Conservation? An Assessment of Livelihood and Environmental Outcomes of Non-Timber Forest Product Trade in Asia, Africa, and Latin America. Ecology and Society 11 (2): 20 [disponible en:http://www.ecologyandsociety. org/vol11/iss2/art20/].

Lambert, D. 1998. Primate Frugivory in Kibale National Park, Uganda, and its Implications for Human Use of Forest Resources. African Journal of Ecology 36: 234-240.

Lawrence, A. 2003. No Forest Without timber? International Forestry Review 5 (2): 87-96.

Lopez, R., y Cavelier, I. 2007. Productos forestales no maderables en los Andes colombianos: una aproximación a su conocimiento y monitoreo. En: Armenteras, D. \& N. Rodríguez (eds.). 2007. Monitoreo de los ecosistemas andinos 1985-2005: Síntesis y perspectivas. Instituto Alexander von Humboldt. Bogotá. Págs. 91-104.

Marshall, E., A. C. Newton \& K. Schreckenberg. 2003. Commercializing Non-Timber Forest Products: First Steps in Analysing the Factors Influencing Success. International Forestry Review 5 (2): 128-137.

Mendoza, A., D. Piñero \& J. Sarukhán. 1987. Effects of Experimental Defoliation on Growth, Reproduction, and Survival of Astrocaryum mexicanum. Journal of Ecology 75: 545-554.

Menges, E. 2000. Population Viability Analyses in Plants: Challenges and Opportunities. Trends in Ecology and Evolution 15: 51-56.

Moegenburg, S. M. \& D. J. Levey. 2002. Prospects for Conserving Biodiversity in Amazonian Extractive Reserves. Ecology Letters 5: 320-324.
Myers, N. 1988. Tropical Forest: Much More than Stocks of Wood. Journal of Tropical Ecology 4: 209-221.

Nakazono, E. M., E. M. Bruna \& R. C. G. Mesquita. 2004. Experimental Harvesting of the Non-Timber Forest Product Ischnosiphon polyphyllus in Central Amazonia. Forest Ecology Management 190: 219-225.

Nault, A. \& D. Gagnon. 1993. Ramet Demography of Allium triccocum, a Spring Ephemeral, Perennial Forest Herb. Journal of Ecology 81: 101-119.

Nantel, P., D. Gagnon \& A. Nault. 1996. Population Viability Analysis of American Ginseng and Wild Leek Harvested in Stochastic Environments. Conservation Biology 10: 608-621.

Ndangalasi, H. J., R. Bitarihob, R. Delali \& B.K. Dovie. 2007. Harvesting of Non-Timber Forest Products and Implications for Conservation in Two Montane Forests of East Africa. Biological Conservation 134: 242-250.

Nepstad, D. C. \& S. Schwartzman. 1992. NonTimber Product Extraction from Tropical Forest: Evaluation of a Conservation and Development Strategy. Advances in Economic Botany 9:VII-XII. New York Botanical Garden. Nueva York.

Neumann,R.P.\&E.Hirsch.2000.Commercialization of Non-Timber Forest Products: Review and Analysis of Resource. CIFOR. Bogor, Indonesia. Pg. 187.

Olmsted, I. \& E. R. Álvarez-Buylla. 1995. Sustainable Harvesting of Tropical Trees: Demography and Matrix Models of Two Palm Species in Mexico. Ecological Applications 5: 484-500.

Padoch, C. 1992. Marketing of Non-Timber Forest Products in Western Amazonia: General Observations and Research Priorities. Advances in Economic Botany 9: 43-50.

Pfab, M. F. \& M.A. Scholes. 2004. Is the Collection of Aloe peglerae from the Wild Sustainable? An Evaluation Using Stochastic Population Modeling. Biological Conservation 118: 695-701. 
Paoli, G. D., D. Peart, M. Leighton \& I. Samsoedins. 2001. An Ecological and Economic Assessment of the Non-Timber Forest Product Gaharu Wood in Gunung Palung Natinal Park, West Kalimantan, Indonesia. Conservation Biology 15 (6): 1721-1732.

Panayotou, T. \& P.S. Ashton. 1992. Not by Timber Alone: Economics and Ecology for Sustaining Tropical Forests. Island Press. Washington. Pg. 275.

Peters, C. M., A. H. Gentry \& R. O. Mendelsohn. 1989. Valuation of an Amazonian Rainforest. Nature 339: 655-656.

Peters, C.M. 1991. Plant Demography and Management of Tropical Forest Resources: A Case Study of Brosimum alicastrum in Mexico. En: Gómez-Pompa, T., C. Whitmore \& M. Hadley (eds). Rainforest Regeneration and Management. UNESCO. París. Págs. 265-272.

Peters, C. M. 1994. Sustainable Harvest of NonTimber Plant Resources in Tropical Moist Forest: An Ecological Primer. Biodiversity Support Program. Washington. Pg. 48 http://159.226.69.10/ whoweare/sustanibility\%20primer.pdf

Peters, C. M. 1996. The Ecology and Management of Non-Timber Forest Resources. World Bank. Washington. World Bank Technical Paper 322. Pg. 157.

Piñol, J. \& J. Martínez-Vilalta. 2006. Ecología con números. Una introducción a la ecología con problemas y ejercicios de simulación. Pg. 419.

Plotkin, M. \& L. Famolare (eds). 1992. Sustainable Harvest and Marketing of Rainforest Products. Conservation International. Island Press. Washington. Pg. 340.

Ratsirarson, J., J.A. Silander \& F. Richard. 1996. Conservation and Management of a Threatened Madagascar Palm Species Neodypsis decaryii. Conservation Biology 10: 40-52.

Redford, K. 1992. The Empty Forest. Bioscience 42 (6): 412-422.

Redford, K. H. \& S. E. Sanderson. 2000. Extracting Humans from Nature. Conservation Biology 14: 1362-1364.
Rodríguez-Buriticá, S., M. A. Orjuela \& G. Galeano. 2005. Demography and Life History of Geonoma orbignyana: An Understory Palm Used as Foliage in Colombia. Forest Ecology and Management 211: 329-340.

Ros-Tonen, M. A. F. \& K. F. Wiersum. 2005. The Scope for Improving Rural Livelihoods Through Non-Timber Forest Products: An Evolving Research Agenda. Forest, Trees and Livelihoods 15: 129-148.

Salafsky, N., F. L. Dugelby \& J. W. Terborgh. 1993. Can Extractive Reserves Save the Rainforest? An Ecological and Socioeconomic Comparison of Non-Timber Forest Product Extraction Systems in Peten, Guatemala, and West Kalimantan, Indonesia. Conservation Biology 7: 39-52.

Salafsky, N. \& E. Wollenberg. 2000. Linking Livelihoods and Conservation: A Conceptual Framework and Scale for Assessing the Integration of Human Needs and Biodiversity. World Development 28 (8): 1421-1438.

Schwartzman, S., A. Moreira \& D. Nepstad. 2000. Rethinking Tropical Forest Conservation: Perils in Parks. Conservation Biology 14: 1351-1357.

SCBD. 2001. Sustainable Management of Non-Timber Forest Resources. CBD Technical Series 6. Secretariat of the Convention on Biological Diversity. Montreal. Pg. 30. http://www.cbd. int/doc/puplications/cbd-ts-06.pdf

Shankar, U., K. S. Murali, R. U. Shaanker, K. N. Ganeshaiah \& K. S. Bawa. 1996. Extraction of Non-Timber Forest Products in the Forest of Bilingiri Rangan Hills, India. III. Productivity, Extraction and Prospects of Sustainable Harvest of Amla Phyllanthus emblica (Euphorbiaceae). Economic Botany 50: 270-279.

Shankar, U., R. Hedge \& K. S. Bawa. 1998. Extraction of Non-Timber Forest Products in the Forest of Bilingiri Rangan Hills, India. VI. Fuelwood Pressure and Management Options. Economic Botany 52: 320-336.

Sheil, D. \& S. Wunder. 2002. The Value of Tropical Forest to Local Communities: Complications, Caveats, and Cautions. Conservation 
Ecology 6 (2): 9. [disponible en: http://www. consecol.org/vol6/iss2/art9].

Siebert, S. 2004. Demographic Effects of Collecting Rattan Can and Their Implications for Sustainable Harvesting. Conservation Biology 18: 424-431.

Sinha, A. \& K. S. Bawa. 2002. Harvesting Techniques, Hemiparasites and Fruit Production in Two Non-Timber Forest Tree Species in South India. Forest Ecology and Management 168: 289-300.

Soehartono, T. \& A. C. Newton. 2001. Conservation and Sustainable Use of Tropical Trees in the Genus Aquilaria II. The Impact of Gaharu Harvesting in Indonesia. Biological Conservation 97: 29-41.

Stewart, K. M. 2001. The Commercial Bark Harvest of the African Cherry (Prunus africana) on Mount Oku, Cameroon: Effects on Traditional Uses and Population Dynamics. Ph. D. Dissertation. Florida International University.

Stewart, K. M. 2003. The African Cherry (Prunus africana): Can Lessons be Learned from an Over-Exploited Medicinal Tree? Journal of Ethnopharmacology 89: 3-13.

Svenning, J. C. 2002. Crown Illumination Limits the Population Growth Rate of a Neotropical Understorey Palm (Geonoma macrostachys, Arecaceae). Plant Ecology 159: 185-199.

Svenning, J. C. y M. J. Macia. 2002. Harvesting of Geonoma macrostachys Mart. Leaves for Thatch: An Exploration of Sustainability. Forest Ecology and Management 167: 251-262.

Ticktin, T. \& T. Johns. 2002. Chinanteco Management of Aechmea magdalenae (Bromeliaceae): Implications for Incorporating TEK and TRM in Management Plans. Economic Botany 56: 43-57.

Ticktin, T., P. Nantel, F. Ramírez \& T. Johns. 2002. Effects of Variation on Harvest Limits for Non-Timber Forest Species in Mexico. Conservation Biology 16: 691-705.

Ticktin, T. 2004. The Ecological Implications of Harvesting Non-Timber Forest Products. Journal of Applied Ecology 41: 11-21.
Ticktin, T. 2005. Applying a Metapopulation Framework to the Management and Conservation of a Non-Timber Forest Species. Forest Ecology and Management 206: 249-261.

Tuljapurkar, S. \& H. Caswell. 1997. StructuredPopulation Models in Marine, Terrestrial, and Freshwater Systems. Chapman and Hall. New York. Cap. 2. Pags. 19-54.

Vallejo, M. I., C. Londoño, R. López, G. Galeano, E. Álvarez \& W. Devia. 2005. Métodos para estudios ecológicos a largo plazo. Establecimiento de parcelas permanentes en bosques de Colombia. Instituto Alexander von Humboldt. Bogotá. Pg. 209.

Vantomme, P., A. Markkula \& R. N. Leslie. 2002. Bon-Wood Forest Products in 15 Countries of Tropical Asia. An Overview. FaO. Bangkok. Pg. 200. http://www.fao.org/docrep/005/ab598c00. htm

Vantomme P. 2007. Global Trends in NwFP Trade and their Prospects for Income Generation. En: International Conference on Sustainable Development of Non-Timber Forest Products and Services. Beijing. Págs. 26-28

Vázquez, R. \& A. H. Gentry. 1989. Use and Misuse of Forest Harvested Fruits in Iquitos Area. Conservation Biology 3: 350-61.

Wong L. G. Jennifer \& T. Kisrti. 2001. Evaluación de los recursos de productos forestales no madereros. Experiencias y principios biométricos. Pg. 124.

Zuidema,P.A.2000.Demography ofExploited Tree Species in the Bolivian Amazon. Ph.D. Thesis. PROMAв Scientific Series 2. Utrecht University. Utrecht, The Netherlands. http://igitur-archive. library.uu.nl/dissertation/1932990/inboud.htm

Zuidema, P. A. \& R. G. Boot. 2002. Demography of the Brazil Nut Tree (Bertholletia excelsa) in the Bolivian Amazon. Impact of Seed Extraction on Recruitment and Population Dynamics. Journal of Tropical Ecology 18: 1-31. 